\title{
On the Dynamic Order of Structured Escherichia coli Growth Models
}

\author{
Bernhard O. Palsson and Abhay Joshi \\ Department of Chemical Engineering, University of Michigan, \\ Ann Arbor, Michigan 48109-2136
}

Accepted for publication September 2, 1986

Reliable dynamic descriptions of cellular growth are important for many practical applications including bioreactor design and control. A chemically structured growth model of Escherichia coli has been formulated and herein we focus on finding the essential dynamic order of the metabolic part of this model. Standard linear analysis is applied and the main finding is that the model contains three essential modes of motion over the time scale of growth. The doubling time is successfully predicted from an unstabie growth motion and the metabolite composition of the three modes of motion suggests that only a three pool metabolic model is necessary. The three pools correspond to important groups of macromolecules; protein, nucleic acids and cell wall constituents.

\section{INTRODUCTION}

Dynamic models of bacterial growth are needed for design and control of bioreactors. Oversimplified descriptions, like the simple Monod growth model that describe the state of the organism with a single variable, have been extensively used. The increasing focus of currently developing biotechnology on intracellular events signifies an impetus for developing physiologically rational growth models that account for the underlying well documented biochemistry. Pioneering steps in this direction have been taken ${ }^{1-3}$ and an exponential growth single-cell model (SCM) for $E$. coli has been formulated. The model consists of detailed description of about $18 \mathrm{key}$ metabolites and it contains over 100 kinetic parameters. However, this degree of complexity is likely to hamper the practical utility of this model. A balance between physiological reality and simplicity is needed to develop tractable models that are of practical utility.

The SCM can serve as a starting point for the development of a simplified but physiologically rational $E$. coli growth model. The important task is to distill out the dynamic essentials from the complex model that are necessary to describe the growth process itself. $A$ priori it seems unlikely that all model variables and kinetic parameters play an important role in deciding the fate of cellular growth.
The following issue is addressed in this paper: given the chemically structured complex model of $E$. coli to what extent is the detailed information contained in the model relevant to the description of $E$. coli growth. Specifically what is the essential dynamic order of the growth process. We will show how judicious application of traditional system science techniques to the SCM accomplishes this goal. We propose a new simple physiologically rational "three pool" model as a compromise between the oversimplified Monod growth model and the complex structured description.

\section{DEVELOPMENT OF A SIMPLE E. coli GROWTH MODEL}

\section{The Structured E. coli Growth Model}

The E. coli SCM visualizes the "whole cell" as an expanding bioreactor that is free to change its size and shape and to respond to changes in external glucose and ammonium concentrations. The SCM has the capacity to predict changes in cell morphology and cellular composition during growth. ${ }^{1-3}$ Growth characteristics such as cell size and shape, length of C and D phases, points of DNA initiation and termination are all natural responses of the cell model.

The complete model of the cell accounts for 18 or more metabolites which represent the inherent dynamic structure of the underlying metabolism. Each equation in this model corresponds to a dynamic mass balance on a particular metabolite. Intermediary metabolism is coupled with a set of another auxiliary description related to cell morphology and decision characteristics of the cell (see Fig. 1). The model equations are too complicated to justify a detailed listing here. Such a listing can be found in the original references ${ }^{2,3}$ though a brief description of the model is given in Figure 1. We are only concerned with the "metabolic" part of this model. We will not examine the auxiliary parts of the model as they are described as explicit functions of the metabolite concentrations. 


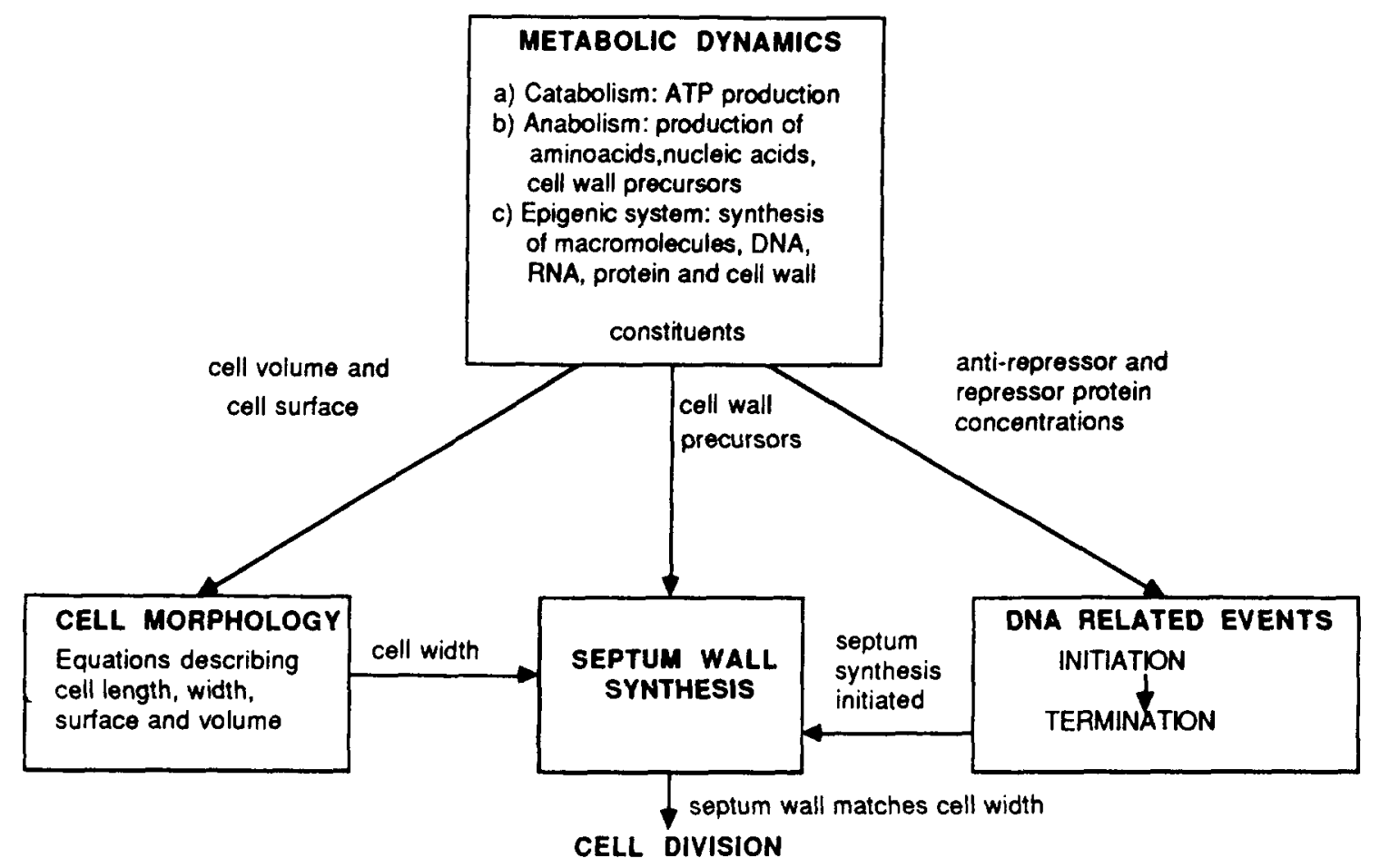

Figure 1. Topological structure of $E$. coli growth model.

\section{Examination of Model Dynamics}

To obtain qualitative information about the dynamics of cell growth process, we linearize a set of kinetic equations around a reference point as:

$$
\frac{d \mathbf{x}}{d t}=f(\mathbf{x})=f\left(\mathbf{x}_{\mathrm{ref}}\right)+\mathbf{J}\left(\mathbf{x}-\mathbf{x}_{\mathrm{ref}}\right)+\cdots
$$

where $\mathbf{x}$ is a vector of concentrations; $f(x)$ is a $n$-dimensional nonlinear function containing the reaction laws; $\mathbf{J}$ is the Jacobian matrix $\left(d f_{i} / d x_{j}\right)$; and the subscript ref denotes the reference condition. The reference point here is a newly born cell.

The Jacobian matrix is then diagonalized $\mathbf{J}=\mathbf{M}^{-1} \mathbf{\Lambda} \mathbf{M}$ where the matrix $\boldsymbol{\Lambda}$ has the eigenvalues of $\mathbf{J}$ on the diagonal and $\mathbf{M}^{-1}$ and $\mathbf{M}$ are matrices comprised of the eigenrows and eigenvectors, respectively. The linearized system is then transformed into a set of dynamically independent variables as:

$$
\frac{d \mathbf{m}}{d t}=\Lambda \mathbf{m}+\mathbf{M}^{-1}\left[f\left(\mathbf{x}_{\mathrm{ref}}\right)-\mathbf{J} \mathbf{x}_{\mathrm{ref}}\right] ; \mathbf{m}=\mathbf{M}^{-1} \mathbf{x}
$$

The new variables $m$ are called modes and they move independently of each other on time scales defined by the corresponding eigenvalue. The negative reciprocal of the eigenvalues are the time constants. We can now think of the linearized system, eq. (2), as a set of $n$ decoupled first order subsystems, i.e., each subsystem evolves in time independently of the others. The information obtained through linear analysis is:
1) the time constants, which give the distribution of time scales inherent in the system; and

2) the modal matrix, $\mathbf{M}^{-1}$, which maps the concentration variables onto the time scales.

Although the modes are abstract mathematical quantities, they lead in particular to elegant physical interpretation when linear analysis is applied to chemical kinetics. ${ }^{4,5}$ The main objective of modal analysis is to decompose large interconnected systems into combination of dynamically independent first-order systems. This decomposition is important when it is desired to focus attention on an important subset of the modes, e.g., in our case the modes that move on time scales which overlap with the time span of growth. The SCM model is, however, nonlinear and that the linearization will only give semiquantitative information about the full nonlinear system. The information obtained, however, gives good insight into model dynamics as we will now discuss.

\section{Time Scale Hierarchy and Modal Structure}

A representative modal matrix and time constants for a newly born $E$. coli cell with a doubling time of $45 \mathrm{~min}$ is shown in Table I. The information displayed in Table I immediately gives important information about the dynamic characteristics of this model.

The first column in Table I shows the inherent time constants in the system and inspection of those reveals 
Table 1. A representative modal matrix of a 45-min cell of $E$. coli $\left(A_{1}\right)$ ammonium ions, $\left(A_{2}\right)$ glucose, $\left(P_{1}\right)$ amino acids, $\left(P_{2}\right)$ ribonucleotides, $\left(P_{3}\right)$ deoxy ribonucleotides, $\left(P_{4}\right)$ cell envelope precursors, $\left(M_{1}\right)$ proteins, $\left(M_{4}\right)$ cell envelope constituents, $\left(M_{5}\right)$ glycogen, $\left(E_{3}\right)$ septation enzyme, (RNA, RNA $_{2}, \mathrm{RNA}_{3}$ ) different forms of RNA. This Modal Matrix is calculated for a freshly born cell with a doubling time of 45 min. Similar information is obtained from the Modal Matrix of slower growing cells.

\begin{tabular}{|c|c|c|c|c|c|c|c|c|c|c|c|c|c|c|}
\hline $\begin{array}{l}\text { Mode } \\
\text { no. }\end{array}$ & $\begin{array}{c}\text { Time } \\
\text { constants }\end{array}$ & $A_{1}$ & $A_{2}$ & $P_{1}$ & $P_{2}$ & $P_{3}$ & $P_{4}$ & $M_{1}$ & $M_{4}$ & $M_{5}$ & $E_{3}$ & $\mathrm{RNA}_{1}$ & $\mathrm{RNA}_{2}$ & $\mathrm{RNA}_{3}$ \\
\hline 1 & $-0.95 \mathrm{~h}$ & 0.5 & & 0.1 & -0.1 & -0.1 & $\underline{1}$ & 0.1 & 1.1 & & 0.7 & -0.1 & -0.1 & -0.1 \\
\hline 2 & & 0.5 & 1.0 & & & & $\overline{0} .2$ & & & & & & & \\
\hline 3 & & 0.3 & & -0.2 & & & 1.0 & & & & & & & \\
\hline 4 & & 1.0 & & -0.1 & & & & & & & & & & \\
\hline 5 & $<\min$ & 1.0 & & & & & & & & & & & & \\
\hline 6 & & 1.0 & & 0.1 & -0.2 & 0.5 & & & & & & & & \\
\hline 7 & & -0.2 & & & 0.1 & 1.0 & & & & & & & & \\
\hline 8 & & 0.8 & & 0.1 & 0.1 & 0.2 & -0.1 & & -0.1 & & & & & \\
\hline 9$)$ & & 1.0 & & 0.2 & 0.4 & 0.6 & -0.2 & & -0.2 & & & -0.4 & 0.1 & 1.0 \\
\hline 10$\}$ & $\mathrm{h}$ & 0.2 & & 0.1 & 0.5 & 0.5 & -1 & 0.1 & -1.1 & -0.1 & & 0.6 & 0.6 & 0.5 \\
\hline 11$\}$ & & 0.5 & & 0.3 & -0.5 & -0.5 & $\overline{1}$ & 0.5 & $\underline{1.1}$ & & 2 & -0.5 & -0.5 & -0.5 \\
\hline 12$\}$ & $>10 \mathrm{~h}$ & & & & & & $-\overline{0} .1$ & & -0.1 & -0.2 & 1.0 & 0.1 & & \\
\hline 135 & & & & & & & 0.1 & & -0.1 & -0.3 & 1.0 & 0.1 & & \\
\hline
\end{tabular}

that the metabolic transients can be divided into three time regimes:

1) Transients on the order of tens of minutes to an hour. These transients comprise the set of modes which characterize the dynamics over the growth time scale and are of key importance here. The eigenvalue associated with the first mode is positive reflecting the exponential growth character of the system. The negative reciprocal of this unstable eigenvalue gives the time constant for growth of the cell. The relationship between the linearized growth time constant (which is the reciprocal of "specific growth rate") and the doubling time of the cell is given by the relation:

$$
t_{\text {double }}=-\ln (2) t_{\text {growth }}
$$

where $t_{\text {growth }}$ is the time constant of the unstable growth mode. This time constant gives an excellent prediction of the cycle time of the cell. Figure 2 shows the trend of the predicted growth rate and the growth rate obtained through the simulation of the full model on a double reciprocal plot of growth rate and substrate concentration. One important point to note here is that the predicted growth rate is obtained only from the initial growth conditions and no full integration of the eighteen equations is involved. Thus, the unstable eigenvalue is a simple way to predict the approximate time a cell takes to double its contents.

Table I shows that the proteins and amino acids move in a fixed ratio on these three time scales and are hence dynamically equivalent. This feature implies the formation of a metabolic pool that is comprised of proteins and amino acids. Similar observations are made for the pools of cell envelope and its constituents and ribonucleotides, deoxy-ribonucleotides, and RNA. This implies that these three key metabolite pools represent the essential behavior of $E$. coli metabolism during growth. Consequently, the whole system can be re- duced to describe the movement of key metabolite pools during the exponential growth phase of the cell. The three metabolic pools are defined as:

pool $_{1}$ : ribonucleotides, deoxy-ribonucleotides, and RNA,

pool $_{2}$ : amino acid and protein, and

pool $_{3}$ : cell envelope precursors and cell envelope constituents.

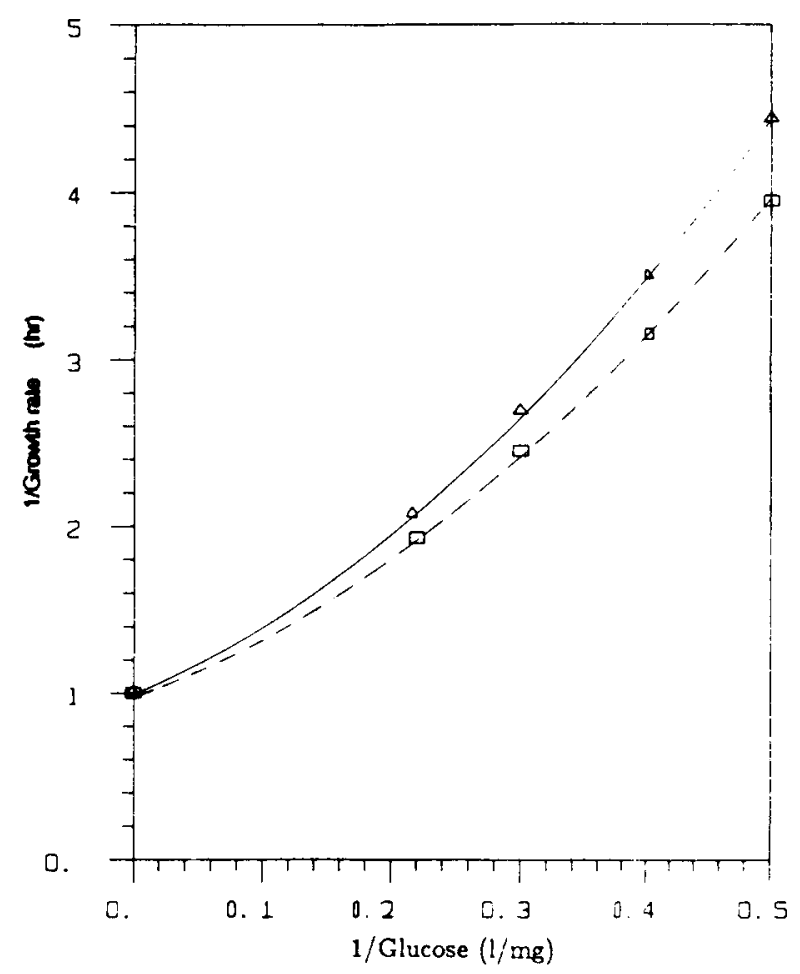

Figure 2. Calculated growth rate as a function of external glucose concentration: $(\triangle)$ predicted growth rate from unstable mode and ( $\square$ ) simulated growth rate from full SCM. The simulated curve presents the chemostat data for $E$. coli $\mathrm{B} / \mathrm{r}-\mathrm{A}$ grown at $37^{\circ} \mathrm{C}$ (ref. 2). 


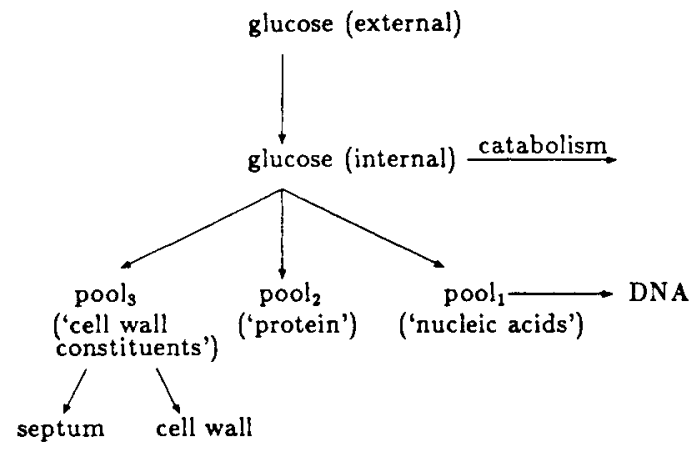

Figure 3. Reduced growth model of $E$. coli.

Similar observations are made when the modal matrices for the $E$. coli cells with cycling times of 0.84 , $1.34,2.18$, and $2.78 \mathrm{~h}$ are calculated.

2) Fast transients (order of seconds to minutes). The modal matrix tells us that the fast transients can be associated with reactions that adjust rapidly to the growth pattern. These transients reprsent low-molecular-weight metabolism. Specifically, they are the following:

a) Modes 2,3, and 4 describe the approach to equilibrium for glucose and cell envelope precursors. Since they move on fast time scales, their dynamics can be relaxed.

b) Mode 5 reveals that ammonium ion concentration is a fast dynamically independent variable and hence its dynamics can be relaxed.

c) Modes 6,7,8, and 9 show the relative magnitude of interaction among the lower molecular weight metabolites. Since these transients are moderately fast they can be relaxed to obtain the adjustment of the low molecular weight metabolites towards the cells growth pattern.

3) Slow transients (on the order of days). The slowest dyanmics in this model represent a slowly changing mass balance on septation enzyme on the order of 15-35 $\mathrm{h}$. This time span is longer than the time scale of interest here.

\section{INTERPRETATION OF GROWTH DYNAMICS}

The simple analysis presented above leads to elegant interpretation of $E$. coli growth dynamics. The events happening on the time scales of ca. $1 \mathrm{~h}$ are essentially those associated with the motion of macromolecules. The interaction between the low-molecular-weight precursors and the macromolecules lead to the formation of three simple metabolic pools that are comprised of macromolecules and their precursors. From these results, one can construct a simple three pool model as shown in Figure 3. The derivation of the full dynamic equations and the rate laws for the simple three pool model is a long procedure and will be presented in a separate, more extensive, communication.

Since the dynamics of cell morphology and certain decision making events are functions of the amount of macromolecules present, i.e., the significant pools, this reduced model would have the ability of simultaneously predicting the cell morphology and cellular composition. Though this view may seem too simplistic, our preliminary simulations indicate that the three pool model do possess the capability of replicating the original model over the growth time scale. These findings along with the full parameter values will be documented in a forthcoming communication.

\section{CONCLUSION}

Reduction in model complexity is essential to make metabolic models attractive for practical use. The results presented herein illustrate the utility of eigenvalue/eigenvector analysis to attain this goal. We see that the dynamic order reduces naturally and logically within the framework of linear analysis to give an insight into the physiological function of key concentration variables.

\section{References}

1. M. L. Shuler, S. Leung, and C. C. Dick, Ann. NY Acad. Sci., 326, 35 (1979).

2. M. M. Domach, S. K. Leung, R. E. Cahn, G. G. Cocks, and M. M. Shuler, Biotechnol. Bioeng., 26, 203 (1984).

3. M. M. Domach, "Refinement and use of a Structured Model of a single cell of Escherichia coli for the description of Ammonia-Limited growth and asynchronous population dynamics," Ph.D. thesis, Cornell University, Ithaca, NY, 1983.

4. B. O. Palsson, "Mathematical Modeling of Dynamics and Control in Metabolic Networks," Ph.D. thesis, University of Wisconsin, Madison, WI, 1984.

5. B. O. Palsson and E. N. Lightfoot, J. Theor. Biol., 111, 273 (1984).

6. J. L. Ingraham, O. Maaloe, and F. C. Neidhart, "Growth of the Bacterial Cell," Sinauer Associates, Inc., Sunderland, MA, 1983. 